\title{
Orbital Angular Momentum Generator Based on Super Smith-Purcell Radiation
}

\author{
Zi-Wen Zhang \\ State Key Laboratory of Advanced \\ Optical Communication Systems and \\ Networks \\ Department of Electronics, Peking \\ University \\ Beijing 100871, China
}

\section{Fan-Hong Li}

State Key Laboratory of Advanced

Optical Communication Systems and

Networks

Department of Electronics, Peking

University

Beijing 100871, China

Liang Zhang

Department of Physics, SUPA

University of Strathclyde

Glasgow G4 0NG, Scotland, UK

\author{
Chao-Hai Du* \\ State Key Laboratory of Advanced \\ Optical Communication Systems and \\ Networks \\ Department of Electronics, Peking \\ University \\ Beijing 100871, China \\ *E-mail: duchaohai@pku.edu.cn \\ $\mathrm{Pu}-\mathrm{Kun} \mathrm{Liu}$ \\ State Key Laboratory of Advanced \\ Optical Communication Systems and \\ Networks \\ Department of Electronics, Peking \\ University \\ Beijing 100871, China
}

\author{
Juan-Feng Zhu \\ State Key Laboratory of Advanced \\ Optical Communication Systems and \\ Networks \\ Department of Electronics, Peking \\ University \\ Beijing 100871, China
}

Adrian W. Cross

Department of Physics, SUPA

University of Strathclyde

Glasgow G4 0NG, Scotland, UK

\begin{abstract}
An orbital angular momentum (OAM) generator based on free-electron-driven super Smith-Purcell radiation (super-SPR) is proposed in this paper. The super-SPR carrying the OAM mode can be generated at a specific angle through interaction between the periodically bunched electron beam and a helical grating. The working frequency and topological charge of the OAM mode is related to the dispersion relation and the operating voltage of the free electron beam. The OAM mode of the topological charge of 1 can be achieved at $320 \mathrm{GHz}$ at the second harmonic. And the field intensity is significantly enhanced compared with the incoherent Smith-Purcell radiation, which finds potential applications in the OAM communications.
\end{abstract}

\section{INTRODUCTION}

Terahertz (THz) communication integrates the advantages of microwave and optical communication, and has many characteristics such as high transmission rate, strong directivity, high security, etc. [1-3]. Orbital angular momentum (OAM), as

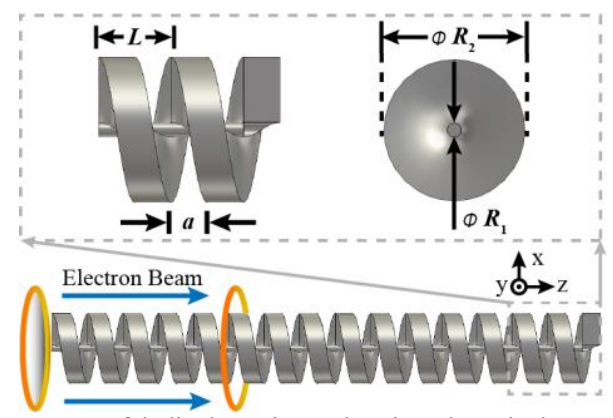

Fig. 1 Structure of helical grating. The ring-shaped electron beam is emitted from left to right, as shown by the blue arrow. a new degree of freedom for electromagnetic waves, is used in communication systems to increase the communication capacity[4, 5]. Currently, OAM waves can be generated using geometric mode conversion methods, spiral phase plates, spatial light modulators, metasurface, and array antennas, etc. [6-9]. However, these approaches still cannot conveniently adjust the topological charge. Hence, the OAM terahertz $(\mathrm{THz})$ source with an adjustable topological charge has been an active area of intense research. Super Smith-Purcell radiation (super-SPR) is a promising solution to achieve this goal. In 1998, J. Urata et al. [10] of Dartmouth University discovered a new radiation phenomenon based on the coherent Smith-Purcell (SP) radiation: super-SPR. When the wave vector of the free electron matches with the wavenumber of spoof surface plasmon polariton (SSP), the SSP mode can be effectively excited. The velocity of the direct current electron beam is modulated during the interaction between the beam-SSP interaction and the bunched electron can be obtained. The bunched electron beam carries the energy of harmonics, and super-SPR is formed when the harmonic frequency is located in the radiative region. And the field intensity of super-SPR is much greater than that of incoherent $\mathrm{SP}$ radiation (ISPR).

In this paper, the OAM radiation is generated by the interaction of the SSP of helical grating and the electron beam. When electron energy is $20 \mathrm{keV}$, the second harmonic superSPR with topological charge of 1 can be carried at $320 \mathrm{GHz}$. The field strength is significantly enhanced compared to the ISPR. 


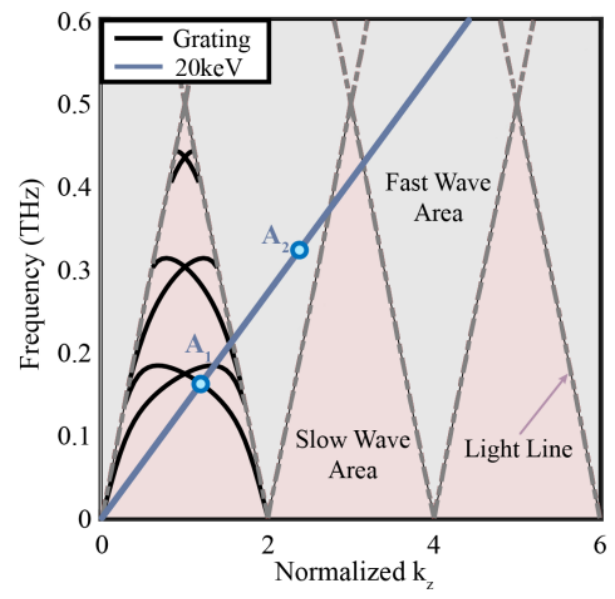

Fig. 2 Dispersion relations of helical grating and electron beam. The black line is the dispersion of the helical grating. The pink and gray region are slow and fast wave areas.

\section{ANALYSIS AND RESULTS}

\section{A. Dispersion of helical grating and electron beam}

The structure of the helical grating is shown in Fig. 1, which is capable of supporting surface plasmon polaritons carrying OAM [11]. The period $L$ of grating is $0.3 \mathrm{~mm}$, the width $a$ of the groove is $0.15 \mathrm{~mm}$, the inner metal rod radius $\mathrm{R}_{1}$ is $0.3 \mathrm{~mm}$ and the outer radius $R_{2}$ is $0.03 \mathrm{~mm}$. Utilizing the finitedifference time-domain method, the dispersion relation of the helical grating is numerically calculated, and is represented by the black line in Fig. 2. The dispersion relation of the electron beam [12] is

$$
\omega=v_{e} k_{z}
$$

which is shown as the blue line in Fig. 2.

\section{B. $U=20 \mathrm{keV}$}

When the energy of free electron is $10 \mathrm{keV}$, the intersection frequency, i.e., the frequency of the intersection point between the dispersion line of free electron and the dispersion line of the fundamental mode of the helical grating, is $160 \mathrm{GHz}$ (the point $\mathrm{A}_{1}$ in the Fig.1). Consequently the DC electron beam is modulated and bunched at a frequency $f_{A 1}=160 \mathrm{GHz}$. Since the second harmonic frequency $f_{A 2}=320 \mathrm{GHz}$ is located in the fast wave region, a second harmonic super-SPR will be generated as shown in Fig. 2, which is validated by the PIC solver in the CST studio. The simulation results are presented in Fig. 3. Compared with the ISPR, the field intensity is enhanced by 5 times while keeping the other parameters unchanged. As shown in the inset of Fig.3 (a), the angle between the radiation direction and the velocity of the electron beam is $55^{\circ}$. The electric field profiles $E_{z}$ at the radiation frequency $f_{A 2}=320 \mathrm{GHz}$ is shown in the inset of Fig. 3 (b), which indicates that the topological charge of the OAM mode is 1 .

\section{CONCLUSION}

In this paper, a convenient method for generating a OAM mode in the THz band based on the super-SPR is proposed. The

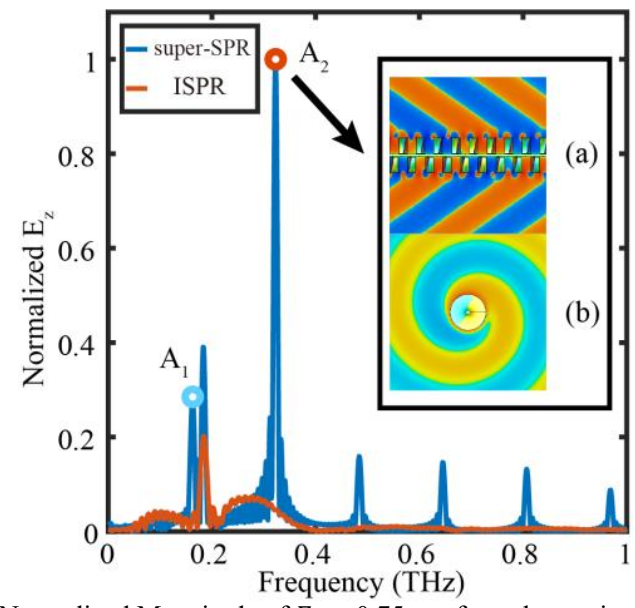

Fig. 3. Normalized Magnitude of $E_{z}$ at $0.75 \mathrm{~mm}$ from the grating surface when the electron beam energy is $20 \mathrm{keV}$. The insect (a) and (b) are the radiation pattern and field distribution of $E_{z}$ at $A_{2}$.

dispersion relation of the spiral grating is calculated by numerical method. When the electron energy is $20 \mathrm{keV}$, the OAM with topological charge of 1 is generated at $320 \mathrm{GHz}$. Compared to incoherent Smith-Purcell radiation, their radiation intensity has been significantly enhanced. The frequency can be tailored by the energy of the free electron, which can be utilized to generate a millimeter wavelength OAM mode in the $\mathrm{THz}$ frequency range. The future plan is to develop compact and tunable $\mathrm{THz}$ OAM communication sources with higher frequency and higher efficiency based on the proposed method.

\section{ACKNOWLEDGMENT}

This work was supported in part by the National Natural Science Foundation of China under contracts 61861130367, 61531002, NSAF-U1830201, and 61971013. It was also supported in part by the Newton Advanced Fellowship from Royal Society (NAF/R1/180121), United Kingdom.

\section{REFERENCES}

T. Nagatsuma, G. Ducournau, and C. C. Renaud, "Advances in terahertz communications accelerated by photonics," Nature Photonics, vol. 10, no. 6, p. 371, 2016.

[2] S. Koenig et al., "Wireless sub-THz communication system with high data rate," Nature photonics, vol. 7, no. 12, p. 977, 2013.

[3] T. Kleine-Ostmann and T. Nagatsuma, "A review on terahertz communications research," Journal of Infrared, Millimeter, and Terahertz Waves, vol. 32, no. 2, pp. 143-171, 2011.

[4] S. Ge et al., "Terahertz vortex beam generator based on a photopatterned large birefringence liquid crystal," Optics express, vol. 25, no. 11, pp. 12349-12356, 2017.

[5] L. Zhu et al., "Experimental demonstration of basic functionalities for $0.1-\mathrm{THz}$ orbital angular momentum (OAM) communications," in OFC 2014, 2014: IEEE, pp. 1-3.

[6] E. Karimi, S. A. Schulz, I. De Leon, H. Qassim, J. Upham, and R. W. Boyd, "Generating optical orbital angular momentum at visible wavelengths using a plasmonic metasurface," Light: Science \& Applications, vol. 3, no. 5, pp. e167-e167, 2014.

[7] A. M. Yao and M. J. Padgett, "Orbital angular momentum: origins, behavior and applications," Advances in Optics and Photonics, vol. 3, no. 2, pp. 161-204, 2011.

[8] M. Uchida and A. Tonomura, "Generation of electron beams carrying orbital angular momentum," nature, vol. 464, no. 7289, pp. 737-739, 2010. 
[9] E. Brasselet, M. Malinauskas, A. Žukauskas, and S. Juodkazis, "Photopolymerized microscopic vortex beam generators: Precise delivery of optical orbital angular momentum," Applied Physics Letters, vol. 97, no. 21, p. 211108, 2010.

[10] J. Urata, M. Goldstein, M. F. Kimmitt, A. Naumov, C. Platt, and J. E. Walsh, "Superradiant Smith-Purcell Emission," Physical Review Letters, vol. 80, no. 3, pp. 516-519, 01/19/ 1998, doi: 10.1103/PhysRevLett.80.516.

[11] F. Rüting, A. Fernández-Domínguez, L. Martín-Moreno, and F. García-Vidal, "Subwavelength chiral surface plasmons that carry tuneable orbital angular momentum," Physical Review B, vol. 86, no. 7, p. 075437, 2012.

[12] J.-F. Zhu, C.-H. Du, F.-H. Li, L.-Y. Bao, and P.-K. Liu, "FreeElectron-Driven Multi-Frequency Terahertz Radiation on a SuperGrating Structure," IEEE Access, vol. 7, pp. 181184-181190, 2019. 